\title{
Opto-mechanical cross-talk reduction in air-holes multicore fibers
}

\author{
Simon Zaslawski ${ }^{\mathrm{a}}$, Yun Fu ${ }^{\mathrm{a}, \mathrm{c}}$, Zhisheng Yanga ${ }^{\mathrm{a}}$, Krzysztof Markiewicz ${ }^{\mathrm{b}}$, Lukasz Szostkiewicz ${ }^{\mathrm{b}}$, Tomasz \\ Nasilowski ${ }^{\mathrm{b}}$ and Luc Thévenaz ${ }^{\mathrm{a}}$ \\ ${ }^{a}$ EPFL Swiss Federal Institute of Technology, Institute of Electrical Engineering, SCI-STI-LT Station 11, CH-1015 Lausanne, Switzerland \\ ${ }^{b}$ InPhoTech sp. z o.o. Oltarzew, ul. Poznańska 400 05-850 Ożarów Mazowiecki \\ ${ }^{c}$ Fiber Optics Research Center, Key Laboratory of Optical Fiber Sensing and Communications (Ministry of Education), University of Electronic \\ Science and Technology of China (UESTC), Chengdu, 611731, China. \\ Corresponding author e-mail: zhisheng.yang @epfl.ch
}

\begin{abstract}
We report on the use of air-holes in multicore fibers to alleviate intercore perturbations induced by forward stimulated Brillouin scattering. Comparisons with conventional multicore fibers show significant improvement in terms of cross-talk reduction. () 2021 The Author(s)
\end{abstract}

\section{Introduction}

Optical fibers have been known for a very long time to be extremely efficient waveguides for electromagnetic radiation. Like any solid material, they also act as acoustic waveguides and are able to support various kinds of vibrational modes. In an optical fiber, three co-propagating waves - two optical, one acoustic - may couple via an interaction known as forward stimulated Brillouin scattering (FSBS). FSBS was studied as early as 1985 [1], where its perturbative action on signals propagating in single-mode fibers was first measured. FSBS stems from the same physical mechanisms as the more familiar backward stimulated Brillouin scattering (SBS), but presents unique features owing to the nature of the acoustic modes involved in the process. Of particular interest here is the spatial occupancy of these modes, which may lead to opto-mechanical cross-talk in multi-core fibers [2]. The coupling occurs because light propagating in a given core may activate transverse acoustic vibrations across the entire fiber cross-section, which will in turn induce perturbations in the form of phase-modulation on light travelling in a different core. The whole process is facilitated by the fact that, unlike the case of backward SBS, no strict-phase matching condition is required $[3,4]$.

In this paper, we report on the use of air-holes in multi-core fibers (MCF) to alleviate FSBS induced cross-talk. The fiber employed here was originally designed and manufactured by Infotech to reduce optical inter-core cross-talk while maintaining compatibility with optical properties given in ITU G.652 recommendation [5]. Optical isolation is achieved by surrounding each core with a series of air-holes, which incidentally modifies the mechanical behavior of this fiber with respect to conventional MCFs. Unlike conventional MCFs, where the doped cores are embedded in a plain silica rod, the elaborated structure of the air-holes MCF is expected to yield acoustic modes exhibiting complex distributions, resulting in poor acousto-optic mode overlap. Although this ought to be confirmed by an in-depth study of the modal behavior of such type of fiber, the experimental results obtained here preliminarily corroborate this statement. In the experiment, core-to-core opto-mechanical cross-talk is evaluated by a Sagnac interferometer aiming at picking up any phase or polarization variations [2] induced in one core of the MCF by a strong activating optical pulse propagating in another core.

\section{Experimental setup}

A simplified version of the experimental layout used is shown in Fig. 1. Two laser sources operating around $1550 \mathrm{~nm}$ and separated by at least $3 \mathrm{~nm}$ are used to generate an activating pulse and a reading signal, respectively. The activating pulse lasts $1 \mu \mathrm{s}$ and is modulated in intensity at a tunable frequency $\mathrm{f}_{\mathrm{FSBS}}$ to induce harmonic acoustic vibrations in the fiber via electrostriction. The use of a $100 \mathrm{~ns}$ reading "pulse" instead of continuous-wave light is preferred as to avoid cavity effects produced by reflections at the fan/in-fan/out of the multi-core fiber. The two pulses are synchronized in time and launched in the fiber with a short delay as to leave time for acoustic waves to sufficiently build up. The polarization controller in the Sagnac loop is adjusted to tune the phase and polarization states of the two optical waves reaching the photodetector in order to maximize the system sensitivity [2]. The filter is set to ensure that no light from the activating pulse reaches the photodetector. This is necessary even though the two pulses propagate in separate cores, since optical cross-talk may become non-negligible due to the strong power unbalance between the activating and the reading pulse. 


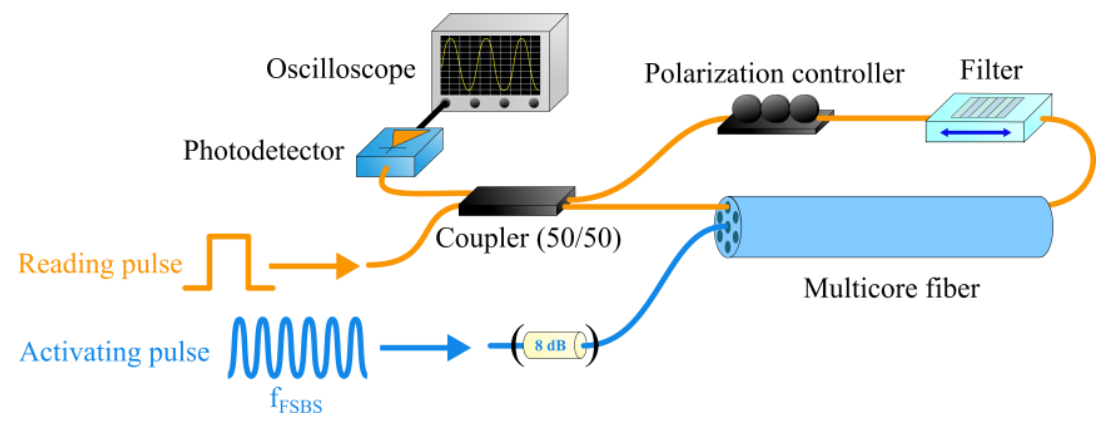

Fig. 1. Experimental layout used to evaluate FSBS induced cross-talk in multicore fibers.

In this paper, one aims at evaluating the performances of air-holes MCFs with respect to conventional MCFs in terms of opto-mechanical cross-talk isolation. The measurements described here above are thus conducted in both types of fibers, namely a $100 \mathrm{~m}$ conventional MCF and a $15 \mathrm{~m}$ hole assisted MCF. For a fair comparison, the activating pulse power $P$ had to be adjusted for each fiber, such that the cumulated effect after propagation in the MCF remains comparable, which is achieved by maintaining the product $P \cdot L$ constant where $L$ is the fiber length. First, the activating pulse peak power $P$ is maximized in the shorter air-holes MCF $(\sim 8 \mathrm{~W})$. The activating pulse is later attenuated by $8 \mathrm{~dB}$ (corresponding roughly to a ratio 15/100) when measuring the conventional MCF.

\section{Experimental evaluation of cross-talks}

The measurements are performed by scanning the activating pulse frequency $\mathrm{f}_{\mathrm{FSBS}}$ between $100 \mathrm{MHz}$ and $900 \mathrm{MHz}$ with a $0.2 \mathrm{MHz}$ scanning step. Thanks to the Sagnac configuration (see Fig. 1), FSBS induced disturbances (either polarization or phase) translate to intensity modulations oscillating at $\mathrm{f}_{\mathrm{FSBS}}$. For each frequency scanned, the perturbed interference pattern is acquired via an oscilloscope and analyzed in the frequency domain by computing its discrete Fourier transform. Since the modulating frequency is known, cross-talk is estimated as the magnitude of the Fourier coefficient closest to $f_{\text {FSBS. The }}$ experimental results are shown in Fig.2 (a), (c) and (e) for the conventional MCF and Fig.2 (b), (d) and (f) for the air-holes MCF. A medallion on each side of the figure shows the cross-section of the corresponding fiber as well as the cores probed during the experiment. The legends indicate which core is driven by the activating pulse and which core is used for reading, e.g. 1 to 2 means activation is done in core number 1 while reading is performed in core number 2. Due to the significant response difference between the two fibers, the black dashed line indicates the corresponding range displayed on the figures from the right-hand side.

The results show significant differences between the two fibers. In the conventional MCF, sharp peaks indicate the presence of strong radial acoustic modes whenever the central core is involved, as evidenced in Fig. 2 (a) and (c). When measuring FSBS induced perturbations from side-core to side-core, one observes a broad spectrum [2], as shown in Fig. 2 (e). We emphasize that the goal pursued in this paper is not to conduct an in-depth analysis of the modal structure of such fiber (see e.g. [2] for a detailed analysis), rather we are interested in detecting any phase or polarization variations induced by opto-mechanical cross-talk. The air-holes MCF displays high opto-mechanical suppression compared to conventional MCF as evidenced in Fig. 2 (b), (d) and (f). The polarization/phase variations picked by our system barely exceed noise level, especially in the case of side-core to side-core cross-talk evaluation (see Fig. 2 (f)). Still, a handful of dominant modes are observable when the central core is involved either for activation or for reading as depicted in Fig. 2 (b) and (d). This confirms the validity of our experimental setup, and the fact that acoustic modes are indeed activated but do not contaminate significantly other cores. In a worst-case scenario, i.e. when considering the strongest mode activated in the central core ( 1 to 2$)$ for each fiber, cross-talk is estimated to be reduced by $\sim 9 \mathrm{~dB}$ in the air-holes MCF with respect to the conventional MCF. Note however that such value has to be considered with great precaution, given the poor signal-to-noise ratio in the case of the air-holes MCF. Our results nevertheless point out that this type of fiber is particularly robust to inter-core opto-mechanical coupling. This is attributed to a poor overlap between the well-known mode-field distribution of the optical waves propagating in each core and the overall complex acoustic modal profile imposed by the structure of the hole supported MCF. 

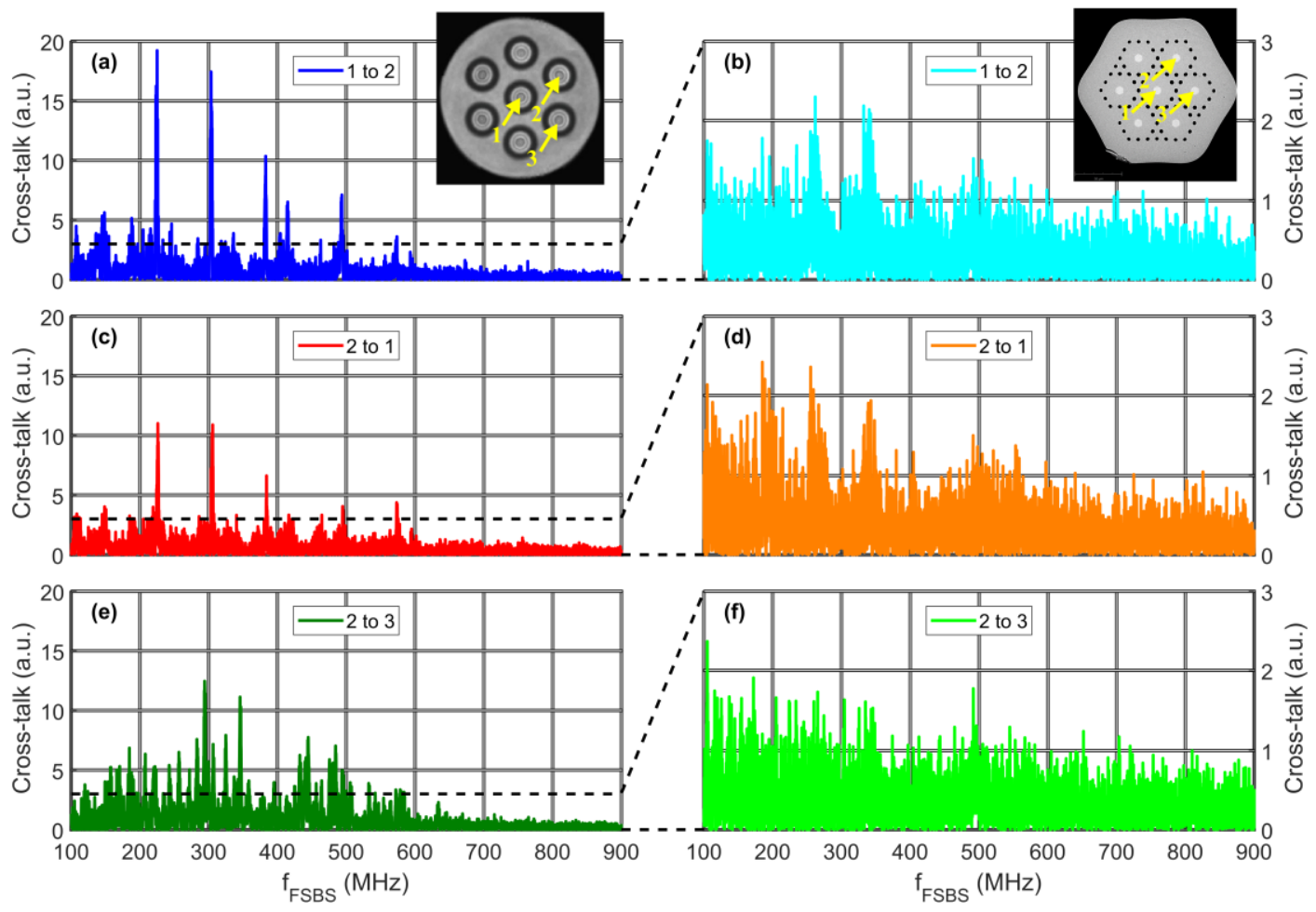

Fig. 2. Core-to-core FSBS perturbation induced in a conventional MCF (a), (c) and (e), and an air-holes MCF (b), (d) and (f). The y-axis unit is arbitrary, and is proportional to the magnitude of the Fourier coefficient at $f_{\text {FSBS}}$. The black dashed line indicate the upper-limit of the $y$-axis in subfigures (b), (d) and (f).

\section{Intra-core perturbations}

A point to be considered is that a good inter-core isolation might come at the expense of stronger intra-core perturbation. The reasoning behind this statement is that the air-holes structure surrounding the fiber cores may act as a local acoustic cavity, hence efficiently sustaining long-lived local acoustic modes. Measurements similar to the ones presented in the previous section are conducted, only this time the two pulses are propagating in the same core. In order to avoid cross-phase modulation [6], the reading pulse follows the strong activating pulse with a delay and picks up perturbations induced by decaying acoustic waves. Notice that in order to couple the two signals in the same port, the introduction of a 50/50 coupler drops the activating pulse power by $3 \mathrm{~dB}$, such that it reaches $\sim 0.6 \mathrm{~W}$ in the $100 \mathrm{~m}$ conventional MCF, and about $4 \mathrm{~W}$ in the $15 \mathrm{~m}$ air-holes MCF. The results are shown in Fig. 3 (a) and (b) for the conventional MCF and the air-holes MCF, respectively.
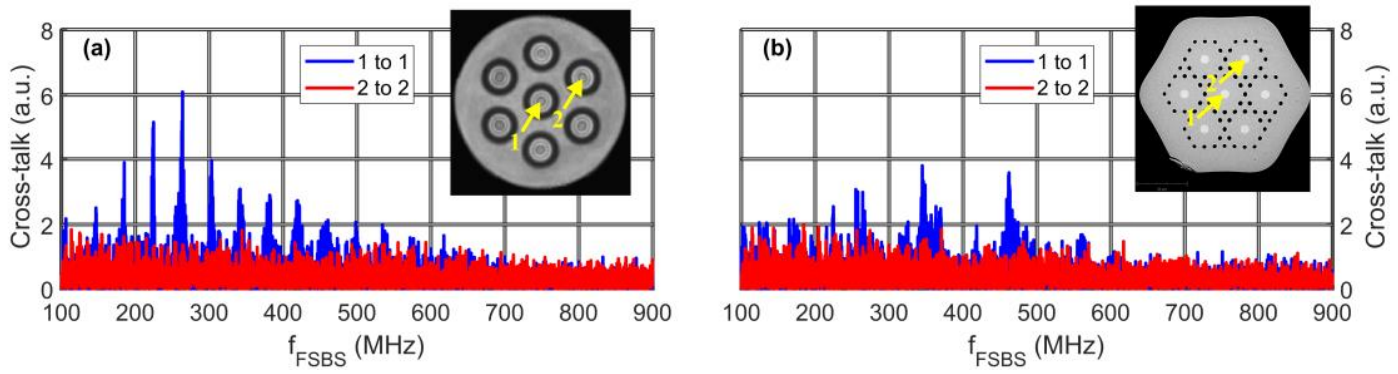

Fig. 3. Intra-core FSBS perturbation induced in a conventional MCF (a) and an air-holes MCF (b). The y-axis unit is arbitrary, and is proportional to the magnitude of the Fourier coefficient at $\mathrm{f}_{\mathrm{FSBS}}$.

This time, both fibers present a distinct modal structure when measuring the central core. The conventional MCF displays a series of sharp resonances corresponding to purely radial modes [2], while the air-holes MCF exhibits somehow a less ordinate structure but still shows few prominent peaks. Fortunately, FSBS does not appear 
stronger in the air-holes MCF than in the conventional MCF, rather it is slightly weaker. Notice that in both cases, FSBS induced perturbations are dominant in the central core when compared to side-cores.

\section{Conclusion}

In this paper, we have investigated the behavior of two types of multi-core fibers in terms of opto-mechanical cross-talk. For each MCF, harmonic acoustic waves were activated by launching a high power intensity modulated pulse in one core, while a different core was inserted in a Sagnac interferometer that picked up any induced phase or polarization change. The results clearly indicate that the benefit of using air-holes to reduce opto-mechanical coupling in MCFs is substantial. Due to their complex architecture, such hole supported MCFs are less propitious to present good overlap between acoustic and optical modes, hence preventing core-to-core opto-mechanical coupling. In addition, better inter-core isolation is not compromised by an enhancement of opto-mechanically induced perturbations when both optical waves are propagating in the same core.

The authors acknowledge the support from the Swiss National Science Foundation (project number: 178895) as well as NODUS project, TEAMTECH programme funded by Foundation for Polish Science.

Y. Fu is supported by the China Scholarship Council (CSC) for her training in EPFL.

\section{References}

[1] R. M. Shelby, M. D. Levenson and P.W. Bayer, “Guided acoustic-wave Brillouin scattering,” Physical ReviewB 31, 5244-5252 (1985).

[2] H. Hagai Diamandi, Yosef London and Avi Zadok, “Opto-mechanical inter-core cross-talk in multi-core fibers,” Optica 4, 289-297 (2017).

[3] M. S. Kang, A. Nazarkin, A. Brenn and P. St. J. Russell, "Tightly trapped acoustic phonons in photonic crystal fibres as highly nonlinear artificial Raman oscillator," Nature Physics 5, 276-280 (2009).

[4] Yair Antman, Alex Clain, Yosef London and Avi Zadok, "Optomechanical sensing of liquids outside standard fibers using forward stimulated Brillouin scattering," Optica 3, 510-516 (2016).

[5] A. Ziolowicz, M. Szymanski, L. Szostkiewicz, T. Tenderenda, M. Napierala, M. Murawski, Z. Holdynski, L. Ostrowski, P. Mergo, K. Poturaj, M. Makara, M. Slowikowski, K. Pawlik, T. Stanczyk, K. Stepien, K. Wysokinski, M. Broczkowska and T. Nasilowski, "Hole-assisted multicore optical fiber for next generation telecom transmission systems," Applied Physics Letters 105, 081106 (2014).

[6] Desmond M. Chow, Zhisheng Yang, Marcelo A. Soto and Luc Thévenaz, "Distributed Forward Brillouin sensor based on local light phase recovery," Nat Comms 9, 2990 (2018). 